Hoffmann, Rudolf Stephan: Todesfall im Verlauf einer Gonorrhöebehandlung. (Gynäkol. Abt., Kaiser Franz-Josetsambulat., Wien.) Zentralbl. f. Gynäkol. Jg. 51, Nr. 14, S. 845-847. 1927.

Nach dem klinischen und pathologisch-anatomischen Befund trat der Tod im angeführten Falle bei einer tripperkranken Frau mit einer gewissen Wahrscheinlichkeit durch Herzlähmung infolge einer im Verlauf der Behandlung plötzlich aufgetretenen Gonokokkensepsis ein. Die im Verlauf der Behandlung verabreichten vier intramuskulären Gonoyatrengaben werden als ursächlich abgelehnt.

Geurg Birnbaum (Würzburg).

Franck, Erwin: Alkoholvergiftung oder Gehirnerschütterung als Ursache plötzliehen Todes. Gutachten aus dem Gebiet der Privatversicherung. (Oberversicherungsamt u. Versorgungsgericht, Berlin.) Ärztl. Sachverst.-Zeit. Jg. 33, Nr. 11, S. 144-146. 1927.

Plötzlicher Tod nach sehr starkem Alkoholgenuß in besonders kalter Nacht bei einem fettreichen 55 jährigen Manne mit Entartung der Coronararterien. Verf. nimmt einen kombinierten Herz-Alkoholtod an, nicht einen Unfalltod infolge Gehirnerschütterung bei Fall von der Treppe. (Pat. wurde am Fuß der Treppe mit dem Kopf nach unten liegend tot vorgefunden.) Der bei der Sektion vorgefundene stark gefüllte Magen sprach gegen Gehirnerschütterung, bei welcher das Erbrechen kaum je vermißt wird. Kurt Mendel (Berlin).。

Stefko, W. H.: Veränderungen in den Gesehleehtsdrüsen bei Unterernährung (und anderen ungünstigen Umwelteinilüssen) mit naehfolgenden konstitutionellen Anomalien. Zeitschr. f. Sexualwiss. Bd. 13, H. 11, S. 345-350. 1927.

Kurze Schilderung der Veränderungen, die Verf. an $\delta$ und $q$ Kindern und Erwachsenen fand, die lange Zeit gehungert hatten und zum Teil an Hunger gestorben waren. Die kindlichen Hoden zeigen eine Atrophie des Keimgewebes bei gleichzeitiger starker Wucherung des Bindegewebes. Bildung und Reifung der Geschlechtszellen hören allmählich auf. Nur einzelne Spermatogonien finden sich in den verengten, nur von einer Zellage ausgelvleideten Samenkanälchen, dagegen vom 12. bis 13. Jahr ab reichlich gruppenweise angeordnete Zwischenzellen. In einigen Fällen zwischen 12 und 16 Jahren konnte Phagocytose der Spermatozoiden durch die Sertoli-Zellen festgestellt werden. In den Hoden der Erwachsenen degenerieren die Zellelemente der Samenkanälchen, die vielfach ohne Epithel gefunden werden. Spermatozoide finden sich in keinem Entwicklungszustand mehr. Das Bindegewebe durchsetzt die Hoden in starken Strängen. In den Ovarien fehlten (in über 120 Fällen zwischen 7 und 40 Jahren) stets die reifen Follikel. Die spärlichen vorhandenen Primordialfollikel lagen woit auseinander in jungem fibroblastischen Bindegewebe, das von der Rinde her eingewuchert war. Auch die interstitielle Drüse war zurückgeblieben. Die Hypoplasie des Genitales (die bei etwa $29 \%$ der russischen Kinder sich findet) geht zumeist mit allgemeinem Infantilismus und allgemeiner Hypoplasie einher, so daB Verf. speziell bei den Frauen die Verbreitung eines neuen ,leptosomen" Konstitutionstypus mit infantilen, eunuchoiden Eigenschaften erwartet als eine Anomalie, die auf dem Umweg über die Geschlechtsdrüsen aus der sozialen Not entspringt.

Risse (Stuttgart).。

\title{
Gerichtliche Geburtshilfe.
}

Gusman, Harry M.: Legal aspects of obstetrics. (Forensische Gesichtspunkte in der Geburtshilfe.). New York state journ. of med. Bd. 26, Nr. 16, S. 705 bis 707. 1926.

Verf. will nur einige wichtige Gesichtspunkte herausgreifen. Es kann sein, daß der ärztliche Gutachter über die Frage der Schwangerschaft gehört wird. Seine Diagnose kann auf Möglichkeit, Wahrscheinlichkeit oder Sicherheit der Gravidität lauten. Möglichkeit liegt vor, wenn die Möglichkeit einer Konzeption gegeben war, wenn ein oder einige subjektive Schwangerschaftszeichen oder ein geringer Grad von Vergrößerung des Leibesumfanges festgestellt werden. Wahrscheinlichkeit liegt vor, wenn diese Zeichen ständig deutlicher werden, und andere Zeichen hinzutreten. Sichere Schwangerschaft liegt vor bei Feststellung kindlicher Herztöne, von Kindesbewegungen, Feststellung des Chadwickschen Zeichens und der uterinen intermittierenden Kontraktionen nach Braxton Hicks. In einzelnen Fällen bei Vergrößerung des Leibesumfanges und Unklarheit der übrigen Symptome empfiehlt sich Untersuchung in Narkose. Für das Gutachten dürfen nur die objektiven, d. h. nicht von der Frau, sondern vom Arzt feststellbaren Zeichen maßgebend sein. Für die ersten 3 Monate sind dies: Vergrößerung des Uterus, Veränderung der Brüste und die Erweichungszeichen, nämlich Erweichung der Cervix, des uterinen Segmentes, des Uterus- 